\title{
EDITORIAL \\ Adult spinal deformity surgery: is there a need for a second attending?
}

\author{
Thomas J. Buell, MD, and Justin S. Smith, MD, PhD \\ Department of Neurosurgery, University of Virginia, Charlottesville, Virginia
}

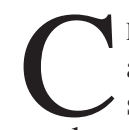
HENG and colleagues share their experience with a single- versus dual-attending strategy for adult spinal deformity (ASD) surgery. ${ }^{1}$ The authors initiated a dual-attending approach to ASD surgery with the combined objectives of improving patient care and fostering collaboration between the neurosurgery and orthopedic departments at their institution. The two surgeons not only aligned operative time for these dual-attending cases, but also aligned their clinic days to co-evaluate patients in order to ensure that both agreed on the management plan and details of surgical treatment. Although residents and/or fellows assisted with organizing the setup and positioning of patients for surgery, the operative procedure was performed by the pair of attending surgeons, who functioned as equal members of the team and operated in parallel. Both surgeons then participated in postoperative care in the hospital and in outpatient follow-up.

Over a period of 2.5 years, the authors performed dualattending surgery for 19 ASD patients. These 19 patients were then matched, based on multiple clinical and surgical parameters, to 19 ASD patients treated surgically by surgeons who did not use a dual-attending approach. The only significant difference in patient outcomes between the dual-attending and single-attending matched groups was that in the group treated by dual attendings a higher percentage of patients felt that their health status was improved at last follow-up, which the authors suggested may have been due to the reassurance patients received by seeing two spine specialists as part of the mutual follow-up strategy. Also notable however, was that at last follow-up the patient groups treated with the dual- or single-attending approach did not have significant differences in estimated blood loss, intraoperative transfusion requirements, length of hospital stay, early complications or reoperations, 90day readmissions, or Oswestry Disability Index or pain scores. The authors concluded that, while their findings support the feasibility of establishing a dual-attending ap- proach, their results do not show objective benefits of dual attendings.

Spinal deformity is highly prevalent in older adults, ${ }^{2}$ and as the global population continues its unprecedented demographic shift toward an older population, the numbers of patients seeking treatment for ASD will markedly increase. A growing number of reported studies have demonstrated the potential for significant improvements in pain and disability with surgery for $\mathrm{ASD},{ }^{3-5}$ but these procedures are complex and the complication rates remain a challenge. ${ }^{6}$ As Cheng and colleagues note, a recent survey of the Scoliosis Research Society found that the majority of surveyed surgeons favored the use of two attendings for ASD surgery for a number of perceived reasons, including reduced complications, decreased operative time, less blood loss, improved outcomes, and less work and stress for the surgeon. ${ }^{7}$ However, most of the surveyed surgeons did not routinely use a dual-attending approach, citing reimbursement challenges for a second surgeon as a major factor.

Many surgeons may perceive potential benefits of a dual-attending approach for ASD surgery, ${ }^{7}$ but it is important to assess these potential benefits in the context of the findings from multiple research studies that have specially focused on this topic. The systematic review provided by Cheng and colleagues does suggest that the use of dual attending surgeons can result in varying degrees of lower intraoperative blood loss and shorter operative time. However, whether there are any differences in complication rates and ultimate clinical outcomes, which are arguably even more important factors, is unclear from the literature, with some studies suggesting benefit and others suggesting no difference. ${ }^{1,8,9}$ Although less easily measured but also important, the use of a dual-attending surgeon approach likely does result in a synergistic reduction in the work and stress faced by each surgeon.

Although the current literature is somewhat mixed with regard to the overall benefits of dual- versus single- 
attending approaches for ASD surgery, it does not appear to demonstrate any negative impact of the use of a dual-attending approach on clinical or operative outcomes. Thus, the question arises, why not routinely use a dual-attending approach for ASD surgery? As Cheng and colleagues note, moving toward a more common use of the dual-attending approach could ultimately have financial consequences, since both surgeons will ultimately expect fair compensation. Surgery for ASD is already among the most costly of surgical procedures. ${ }^{10}$ The added expense of a second attending surgeon, especially if extended beyond the current limited allowances for dual-surgeon reimbursement, could meet a significant challenge from payers.

Perhaps the greatest argument against routine use of dual attending surgeons for ASD surgery is the need to train residents and fellows. Cheng and colleagues allowed residents to be involved in the setup and positioning of the patient and indicated that all team members, including the trainees, were satisfied with their involvement. ${ }^{1}$ Given the low $(7.5 \%)$ percentage of dual-attending cases during the 2.5-year study period, it remains unclear how trainees may have responded if subjected to higher rates of dualattending cases, and consequently, less operative autonomy. Current literature on surgery education cites potential negative consequences from decreasing resident operative autonomy, ${ }^{11}$ which is an important consideration that may limit widespread acceptance of the dual-attending strategy for ASD surgery. These are the same residents and fellows who will be expected to perform these cases in a population that seems to only increase in complexity, with more previous spine surgeries, older age, and increased comorbidities. While the rare savant may sufficiently learn from simply watching the master surgeon, the reality is that, as it continues to be for attending surgeons throughout medical practice, hands-on experience with progressively more challenging cases is the training ground through which surgical skills are sharpened, safety is enhanced, and outcomes are optimized.

An ASD surgeon certainly warrants an assistant who is useful and safe. For some practice situations and for some particularly complex procedures it may be that another attending is the best option, and for these cases both surgeons should be fairly compensated. However, in settings that include residents and fellows, the desire to seek a second attending for ASD surgery should be balanced with the availability of fellows and sufficiently trained senior residents and the obligation to train the next generation to meet the needs of future patients.

https://thejns.org/doi/abs/10.3171/2020.4.SPINE20465

\section{References}

1. Cheng I, Stienen MN, Medress ZA, et al. Single- versus dualattending strategy for spinal deformity surgery: 2-year experience and systematic review of the literature. J Neurosurg Spine. Published online July 10, 2020. doi:10.3171/2020.3. SPINE2016

2. Schwab F, Dubey A, Gamez L, et al. Adult scoliosis: prevalence, SF-36, and nutritional parameters in an elderly volunteer population. Spine (Phila Pa 1976). 2005;30(9): 1082-1085.

3. Acaroglu E, Yavuz AC, Guler UO, et al. A decision analysis to identify the ideal treatment for adult spinal deformity: is surgery better than non-surgical treatment in improving health-related quality of life and decreasing the disease burden? Eur Spine J. 2016;25(8):2390-2400.

4. Kelly MP, Lurie JD, Yanik EL, et al. Operative versus nonoperative treatment for adult symptomatic lumbar scoliosis. $J$ Bone Joint Surg Am. 2019;101(4):338-352.

5. Smith JS, Lafage V, Shaffrey CI, et al. Outcomes of operative and nonoperative treatment for adult spinal deformity: a prospective, multicenter, propensity-matched cohort assessment with minimum 2-year follow-up. Neurosurgery. 2016;78(6): 851-861.

6. Smith JS, Klineberg E, Lafage V, et al. Prospective multicenter assessment of perioperative and minimum 2-year postoperative complication rates associated with adult spinal deformity surgery. J Neurosurg Spine. 2016;25(1):1-14.

7. Scheer JK, Sethi RK, Hey LA, et al. Results of the 2015 Scoliosis Research Society survey on single versus dual attending surgeon approach for adult spinal deformity surgery. Spine (Phila Pa 1976). 2017;42(12):932-942.

8. Ames CP, Barry JJ, Keshavarzi S, et al. Perioperative outcomes and complications of pedicle subtraction osteotomy in cases with single versus two attending surgeons. Spine Deform. 2013;1(1):51-58.

9. Gomez JA, Lafage V, Scuibba DM, et al. Adult scoliosis deformity surgery: comparison of outcomes between one versus two attending surgeons. Spine (Phila Pa 1976). 2017; 42(13):992-998.

10. McCarthy IM, Hostin RA, Ames CP, et al. Total hospital costs of surgical treatment for adult spinal deformity: an extended follow-up study. Spine J. 2014;14(10):2326-2333.

11. Meyerson SL, Teitelbaum EN, George BC, et al. Defining the autonomy gap: when expectations do not meet reality in the operating room. J Surg Educ. 2014;71(6):e64-e72.

\section{Disclosures}

Dr. Smith reports being a consultant for and receiving royalties from Zimmer Biomet and NuVasive; being a consultant for and receiving study group research grants from DePuy Synthes; being a consultant for Stryker and Carlsmed; having stock ownership in Alphatec; receiving fellowship funding and research support from AOSpine; and receiving fellowship funding from the Neurosurgery Research and Education Foundation (NREF).

\section{Correspondence}

Justin S. Smith: jss7f@virginia.edu.

\section{INCLUDE WHEN CITING}

Published online July 10, 2020; DOI: 10.3171/2020.4.SPINE20465.

\section{Response}

\section{Zachary A. Medress, MD, ${ }^{1}$ Sariah Khormaee, MD, PhD, ${ }^{2}$ Martin N. Stienen, MD, FEBNS, ${ }^{1,3,4}$ Anand Veeravagu, MD, ${ }^{1}$ and Ivan Cheng, MD²}

Departments of ${ }^{1}$ Neurosurgery and ${ }^{2}$ Orthopedic Surgery, Stanford University Hospitals and Clinics, Stanford, California; ${ }^{3}$ Department of Neurosurgery, University Hospital Zurich; and ${ }^{4} \mathrm{Clinical}$ Neuroscience Center, University of Zurich, Switzerland

We appreciate the interest of Drs. Buell and Smith in our recent article "Single- versus dual-attending strategy for spinal deformity surgery: 2-year experience and systematic review of the literature." The authors raise several valid points in their letter, including the need to establish objective benefits and minimize drawbacks of a dual-attending model, and perhaps most importantly, Drs. 
Buell and Smith raise concerns about what impact a dualattending model may have on training the next generation of spine surgeons.

The potential impact of a dual-attending model on resident and fellow training in adult spinal deformity operations should be taken very seriously. ${ }^{1-3}$ We agree with the authors that hands-on intraoperative experience remains absolutely critical for surgical education, and that the use of two attendings should be carefully evaluated for its impact on the training experience. From a trainee perspective, much benefit can be derived from exposure to diverse surgical techniques, intraoperative tools, and clinical decision-making. The inclusion of two attendings from different specialties may allow trainees to broaden their exposure to different skill sets if there are significant differences in the working styles between the two specialties in their training institution. It is hypothesized that neurosurgeons become familiar with the routine use of computer-assisted surgical navigation during their cranial operative training and therefore may be more likely to use this navigation method for instrumented spine surgery, as was shown in a survey by Härtl et al. ${ }^{4}$ Accordingly, the dual-attending strategy at Stanford offers orthopedic residents and fellows an opportunity to become more familiar with navigated spine surgery and learn to appreciate its advantages within an established and efficient workflow. On the other hand, neurosurgery residents and fellows can pick up technical pearls from the orthopedic attending for various techniques, such as orthopedic osteotomy operating methods, management algorithms, and principles of systemic bony pathology. As we outlined in the article, the inclusion of two attendings allowed both surgeons to move efficiently through portions of the operation, such as exposure of bony landmarks and placement of pedicle screws. If advanced trainees with established competence participate, resident and fellow involvement can focus on key complex portions of the case, such as three-column osteotomies. For more junior trainees, the workflow may be switched, so that two attendings can do the more advanced sections of the case together as junior trainees work on refining exposure and instrumentation placement.

We appreciate the comments from Drs. Buell and Smith and believe that the dual-attending model offers benefits for trainee education when thoughtfully considered, and that trainee experience is a critical concern that surgeons should ensure is made a high priority.

\section{References}

1. Stienen MN, Bartek J Jr, Czabanka MA, et al. Neurosurgical procedures performed during residency in Europe-preliminary numbers and time trends. Acta Neurochir (Wien). 2019; 161(5):843-853.

2. Stienen MN, Netuka D, Demetriades AK, et al. Neurosurgical resident education in Europe-results of a multinational survey. Acta Neurochir (Wien). 2016;158(1):3-15.

3. Yaeger KA, Munich SA, Byrne RW, Germano IM. Trends in United States neurosurgery residency education and training over the last decade (2009-2019). Neurosurg Focus. 2020; 48(3):E6.

4. Härtl R, Lam KS, Wang J, et al. Worldwide survey on the use of navigation in spine surgery. World Neurosurg. 2013; 79(1):162-172.

INCLUDE WHEN CITING

Published online July 10, 2020; DOI: 10.3171/2020.4.SPINE20512. 